\title{
Étudier la violence homophobe d'un point de vue anthropologique
}

\author{
Réflexions sur la méthodologie du terrain
}

Lia Viola ${ }^{1}$

\begin{abstract}
[Résumé] L'article est une analyse des enjeux liés au positionnement du chercheur, dans le contexte d'une recherche de doctorat sur la violence homophobe dans une ville africaine à majorité musulmane sunnite. Les différents niveaux de l'identité personnelle de l'auteur - provenance géopolitique, genre et orientation sexuelle - sont mis en rapport avec le contexte du terrain, le sujet de recherche et les choix politiques faits. Le récit suit les changements du terrain jusqu'à la description et l'analyse de certains épisodes de violence subis par la chercheuse. La violence potentielle ou effectivement subie est analysée comme le résultat des tentatives de contrôle et des jugements et donc comme une riche source méthodologique de travail. Les notions de visibilité et d'invisibilité sont utilisées de manière réflexive dans le but de comprendre comment la chercheuse peut utiliser son positionnement pour construire son réseau de terrain. En même temps, l'étude veut dénouer les enjeux liés aux choix du chercheur de s'engager politiquement dans la lutte pour les droits des minorités sexuelles. Ce choix a eu des conséquences inattendues, qui n'ont pas été seulement négatives. Dans ce sens les relations conflictuelles avec certains interlocuteurs de terrain sont considérées comme une partie très significative de l'enquête : elles constituent un véritable moyen pour ouvrir de nouvelles pistes d'analyse inattendues. Mots-clés : homophobie, Afrique, violence, positionnement, activisme politique.
\end{abstract}

[Abstract] This article is an analysis of the issues connected with the researcher's position during a PhD fieldwork regarding homophobic violence in an African city with a predominantly Sunni Muslim population. Different levels of the researcher's own identity - geopolitical provenance, gender and sexual orientation - are analyzed in relationship to the context of the fieldwork, the topic of the research, and the political choices of the author. The narration follows the changes of the fieldwork, including the description and analysis of certain violent episodes lived by the researcher. This violence is analyzed as the result of attempts to control and judgements, and as an important source of methodological potential. The notions of visibility and invisibility are utilized in a reflexive way to understand how the researcher can use her own placement to build her fieldwork network. This article also analyzes the choices made by the researcher to develop a political engagement in the fieldwork. Such choices had unpredictable - and not only negative - consequences. Such conflictual situations became an important part of the research conducted, and are considered as a fruitful way to reflect on the issues addressed by the fieldwork.

Keywords: homophobia, Africa, violence, placement, political activism.

\footnotetext{
${ }^{1}$ Université de Turin.
} 


\section{Introduction}

Pendant mon terrain de doctorat sur la violence homophobe ${ }^{2}$ dans une ville africaine ${ }^{3}$ à majorité musulmane sunnite, je me suis confrontée au problème du positionnement du chercheur, tant d'un point de vue méthodologique qu'éthique (et, clairement, politique). La région où j'ai conduit ma recherche est sévèrement touchée par la violence homo-lesbo-transphobe, à la fois physique et psychologique. Avec l'expression " violence physique », nous entendons tous les cas de violence sexuelle, les agressions, les tentatives de meurtre et, entre autres, les homicides. Bien que les homicides soient une minorité d'un point de vue quantitatif, il faut souligner qu'ils produisent et rappellent clairement un jeu de symboles angoissants, très efficaces au niveau collectif. Dans ce contexte on peut constater une verbalisation de l'homophobie au travers du vocabulaire religieux mais, à mon avis, les raisons réelles qui suscitent la violence homophobe sont à chercher dans la construction historique du système hétéronormatif local, auquel ne fait que participer le religieux, et non dans le système symbolique religieux lui-même.

Dans cette analyse, j'essayerai de maintenir l'attention sur le rapport entre les trois niveaux de violence homophobe différents, mais complémentaires : structurel, individuel, et collectif. Je soutiens l'idée que l'homophobie est toujours légitimée et produite par un système structurel hétéronormatif ${ }^{4}$ et cissexiste ${ }^{5}$, qui est lui-même violent et producteur de violence. La définition classique d'homophobie pose l'attention sur la peur irrationnelle (Weinberg, 1972) : notamment, la « phobie » des homosexuels. Même s'il est important de considérer que la violence contre les homosexuels est souvent motivée par une sorte de réaction phobique, j'estime qu'il est important de ne pas la réduire à un niveau individuel. D'un point de vue politique et conceptuel, la conséquence la plus dangereuse de ce type d'analyse s'avère être la dissimulation des mécanismes de violence structurelle et symbolique tout autant au fondement de la discrimination que de l'utilisation de la violence physique.

Des catégories d'analyse différentes ont été proposées dans la littérature scientifique pour décrire et étudier la relation entre le niveau social et le niveau individuel.

\footnotetext{
${ }^{2}$ Avec le terme violence homophobe j'inclus ici tous les cas de violence physique et psychologique motivée par la haine des personnes qui ont des rapports sexuels avec des personnes du même sexe ou qui ont une autodéfinition en tant que personnes homosexuelles.

${ }^{3}$ En raison des dangers auxquels sont toujours exposés mes interlocuteurs, dans cet article je n'expliciterai pas plus le lieu où j'ai conduit mon terrain.

${ }^{4}$ L'hétéro-normativité est un construit social normatif qui définit l'hétérosexualité comme naturelle et donc, d'une certaine façon, obligatoire et donnée.

${ }^{5}$ Le terme cis est la catégorie opposée à trans et inclut donc tous les sujets qui ont une perception de leur propre genre en lien avec le sexe qui leur est attribué à la naissance.
} 
Gregory Herek, par exemple, a introduit le terme heterosexism qui permet d'échapper à l'individualisme lié au concept classique d'homophobie et d'analyser plutôt le niveau macro des idéologies culturelles (Herek, 2000).

Au-delà du débat sur la terminologie utilisée, le sujet principal de ma recherche de doctorat était une analyse de la violence à la fois individuelle et collective en relation avec la structure idéologique hégémonique qui est notamment réglée par la volonté de maintenir les hiérarchies sociales entre les genres, l'hégémonie masculine (Connell, Messerschmidt, 2005) et le système sexe-genre local (Rubin, 2006). Cette structure symbolique du pouvoir entre les genres utilise la violence, ou la menace d'agression, pour discipliner les corps en les enfermant dans des catégories et des stéréotypes de genres, ainsi qu'en moralisant la sexualité et en inhibant les désirs non normatifs.

D'un point de vue ethnographique, ma thématique de recherche était donc analysable au prisme de deux principaux sujets sociaux - les « acteurs de la violence » et les "victimes de la violence», et ce alors que la structure symbolique hétéro-cissexiste apparait comme cadre social. Cette simplification dichotomique entre victimes et exécuteurs de la violence - loin d'être un miroir réaliste de l'interaction sociale - est uniquement fonctionnelle comme point de départ pour la conceptualisation de l'enquête de terrain.

L'un des points cruciaux de ma recherche consistait à comprendre pourquoi certaines personnes décident - dans ce contexte - d'agir violemment contre les homosexuels. Probablement - comme l'a soutenu Judith Butler (2004) - la volonté de tuer (pour analyser les cas les plus éclatants) naît de l'idée que la seule présence de personnes qui ont une orientation sexuelle ou un genre qui ne rentre pas dans la norme définie peut menacer le sens du monde et la vie à part entière. Si donc la violence individuelle est un produit de l'acculturation hétéro-cissexiste, notre analyse doit se concentrer sur la façon dont la violence se construit en lien avec des structures de signification et de sens (Taussig, 1986). L'acte violent peut être conceptualisé comme une forme de communication entre sujets. Il faut donc considérer le rapport victime/bourreaux dans une perspective dialectique qui appréhende la violence comme un langage symbolique disciplinant, exerçant le pouvoir et, d'une certaine façon, défensif.

De plus, le phénomène de «l'homophobie intériorisée $e^{6}$ montre bien qu'un sujet peut éprouver lui-même à la fois le désir homosexuel et être agit par le mécanisme disciplinant et répressif de l'homophobie. Il faut aussi souligner que ma recherche a montré que certains épisodes de violence homophobe surgissent dans le cadre d'un

\footnotetext{
${ }^{6}$ L'homophobie intériorise l'ensemble des sentiments négatifs (comme l'anxiété, le mépris ou l'aversion) éprouvés par certaines personnes homosexuelles envers l'homosexualité des autres ou leurs propres désirs homosexuels.
} 
rapport affectif et sexuel entre deux personnes du même sexe biologique. Il est plutôt difficile d'établir si cette violence est motivée par une forme d'homophobie intériorisée ou par la peur de la visibilité et du jugement social (ou par les deux en même temps). Mais en tenant compte de la violence inhérente aux relations amoureuses/sexuelles, la nécessité d'interroger ethnographiquement le problème du rapport entre homosexuels et homophobes, victimes et bourreaux, s'impose. En partant de cette problématique, la recherche se concentre sur l'étude de la relation complémentaire et interdépendante entre intimité et violence, attraction et répulsion, désir et haine. Il faut pourtant préciser de quelle façon peut être conduite une recherche ethnographique sur la relation entre homosexuels et homophobes, avec le but de comprendre les raisons et les mécanismes à l'origine de l'éclatement de la violence tout en ne s'exposant pas à une situation trop dangereuse.

Dans le développement de cette analyse, il est particulièrement important de ne pas sous-estimer le côté politique et la valeur sociale des mobilisations collectives. Comme l'a souligné Christophe Broqua (2015), les deux mouvements, celui en opposition et celui en faveur des homosexuels, doivent être étudiés en tenant compte de leur interdépendance et de leur relation avec l'Occident. Ce dernier point, et en particulier le problème de la perception locale de la rhétorique occidentale sur les droits de l'Homme, sera un point d'analyse particulièrement important pour bâtir la thèse de cet article. De plus, il est important de considérer qu'une partie de l'islamophobie occidentale trouve sa verbalisation sur la question des droits des minorités sexuelles (Puar, 2007), et peut donc avoir l'effet de renforcer certains mécanismes d'opposition identitaire. En premier lieu néanmoins, il convient de s'interroger sur la façon dont l'homophobie - en tant que violence individuelle tout autant que mobilisation collective - trouve ses mécanismes générateurs dans la relation entre ces opposés. L'ethnographie gagne ainsi à interroger cette relation.

Dans cet article, j'analyserai donc d'un point de vue réflexif mon travail de terrain, en particulier en me focalisant sur les rapports entre la thématique de recherche et sa visibilité, le placement du chercheur et l'omniprésence de la violence. Ceci constitue un point important de l'article : pendant le développement de la recherche, la violence n'était pas conceptualisée comme un sujet analysable d'un point de vue détaché, mais plutôt comme un réseau de symboles à analyser de l'intérieur. Les réflexions recueillies dans cet article sont, donc, la synthèse d'un parcours qui s'est développé en différentes phases. Mon travail se concentrera sur une analyse de certaines variables de mon identité (genre, provenance géopolitique et orientation sexuelle) qui ont influencé mon positionnement, ma relation avec mes interlocuteurs et l'ensemble de ma recherche en général. Ces variables doivent être aussi étudiées en rapport avec le choix de prendre part ou, au contraire, de rester à l'écart de l'activisme LGBTIQ ${ }^{7}$ local.

${ }^{7}$ Acronyme de « lesbien, gay, bisexuel, transgenre, intersexe ou queer ». 
Bien entendu, le genre et la provenance géopolitique sont des variables impossibles à cacher aux yeux des interlocuteurs sur le terrain. Ceci nous oblige à réfléchir sur les symboles provenant de l'Europe et du monde occidental, ainsi que sur les stéréotypes et les catégories de genre. Comme l'a souligné l'anthropologie féministe (Abu-Lughod, 2006), la réflexion sur sa propre identité de genre est fondamentale avant de démarrer une recherche sur le terrain. Il faut également souligner que l'orientation sexuelle est une variable jouant un rôle dans la création des mécanismes d'inclusion ou d'exclusion du chercheur lui-même. À la différence du genre et de la provenance géopolitique, l'orientation sexuelle peut être cachée. C'est-à-dire que le chercheur peut agir intentionnellement en décidant, de manière stratégique, de révéler ou pas (et à qui) son orientation sexuelle. Ces réflexions s'avèrent davantage lourdes de sens pour les chercheurs qui travaillent sur la sexualité et pour les chercheurs qui ne sont pas hétérosexuels. En effet, les systèmes hétéronormatifs sont construits autour du présupposé selon lequel le monde entier serait «naturellement » hétérosexuel et que les personnes homosexuelles et bisexuelles représenteraient une catégorie à part. Les chercheurs homosexuels qui conduisent des terrains dans des lieux où la violence homophobe est particulièrement frappante (et/ou l'homosexualité est criminalisée) doivent décider d'être, ou non, visibles en tant qu'« homosexuels ». Dans le cas de mon terrain, ce choix interagit avec la thématique de recherche : si d'un côté, révéler une orientation sexuelle non hétéronormative peut être utile au niveau méthodologique, surtout dans le rapport avec les interlocuteurs homosexuels, d'autre part cela peut se révéler très dangereux au regard du risque de devenir une cible de violence homophobe.

Par ailleurs, la violence en tant que sujet à étudier et comme danger auquel échapper, élargit l'analyse à des questionnements éthiques et en particulier, à la thématique de l'engagement politique. Cet article est construit atour de mon choix de devenir militante sur le terrain, choix que j'ai fait après quelques mois de recherche en conséquence d'une série d'événements inattendus. Ce choix est donc mis en relation avec d'autres variables de mon identité pour analyser les réactions, les jugements et les tentatives de contrôle provoqués par ma présence et mes actions.

\section{Premiers choix méthodologiques}

Pour focaliser ma recherche sur la relation entre homosexualité et homophobie, j'avais initialement décidé de partager l'ethnographie en deux parties: la première concernait les militants LGBTIQ de la ville et l'autre se penchait sur les leaders de la rhétorique homophobe. Je synthétise avec l'expression «leader de l'homophobie » des personnes qui ont un rôle dans la production du discours homophobe. Souvent ces personnes sont aussi des leaders religieux et donc des leaders communautaires mais, comme je l'ai déjà souligné la construction de la rhétorique homophobe ne peut pas être analysée comme le seul produit du discours religieux. Ce dernier est souvent le 
moyen au travers duquel cette rhétorique trouve sa verbalisation. Il convient aussi de souligner que les militants LGBTI conduisent une lutte clandestine, par conséquent ma relation avec eux était, le plus possible, cachée aux yeux du reste de la communauté citadine. Avec les activistes, j'étais transparente à propos de mon orientation sexuelle et sur le vrai thème de ma recherche. La décision de partager avec eux la vérité sur mon homosexualité était, d'une certaine manière, « utile» d'un point de vue méthodologique : cela m’a aidée à travailler avec empathie.

À l'inverse, avec la population hétérosexuelle de la ville j’ai mis en scène une « fiction d'hétérosexualité » destinée à me préserver de l'exposition à la violence homophobe, et à me permettre de conduire la recherche loin des processus de stigmatisation liés à la représentation, très développée, de l'homosexualité comme «maladie des Occidentaux ». De plus, avec la majorité de mes interlocuteurs, je n'étais pas du tout claire concernant le vrai sujet d'enquête : officiellement ma recherche portait sur les transformations sociales de la ville avec une attention particulière aux questions de genre. Il fut plutôt facile de déplacer mes conversations sur la thématique de l'homosexualité parce qu'elle est considérée - par la majorité des personnes - comme une des pollutions ramenées en Afrique par les Occidentaux. De la même manière, l'activisme pour les droits des minorités sexuelles est vu comme une conséquence des processus néo-colonialistes (Hoad, 2007). La lutte pour les droits des minorités sexuelles s'avère beaucoup plus complexe par rapport aux simplifications faites par les leaders homophobes et à la rhétorique universaliste propre à une partie du mouvement LGBTI occidental.

Pendant ma recherche j'ai analysé, plutôt, comment la violence homophobe croissante est partialement liée à la problématique de la catégorisation des comportements et des identités sexuelles. Il n'y a évidemment aucun doute - cela est en effet démontré par l'ethnologie et l'histoire - concernant l'existence de relations sexuelles entre personnes du même sexe biologique en Afrique avant même le début de la colonisation (Epprecht, 2008; Evans-Pritchard, 1970; Hoad 2007; Morgan, Wieringa, 2005 ; Murray, Roscoe, 1998). Traditionnellement dans le contexte de mon terrain, ces relations n'étaient pas catégorisées en référence au concept occidental d'identité homosexuelle ${ }^{8}$. L'évolution plus récente d'une partie du mouvement LGBTI occidental et son internationalisation sont motivés, à partir des années 1990, par un paradigme politique universaliste visant à s'opposer à l'homophobie dans le monde entier (Massad, 2007) et qui ne s'est uniquement développé grâce à de grandes associations transnationales. En parallèle, les phénomènes du pink-washing et de l'homo-nationalisme (Puar, 2007) ont amené beaucoup de pays, de leaders de gouvernement, d'associations pour les droits de l'Homme et d'activistes LGBTI occidentaux à développer une auto-

8 Pour une analyse critique des concepts d'identité du genre et de la sexualité, voir Weeks (1987) et Butler (1990). 
représentation de l'Occident comme une sorte de paradis gay-friendly où les droits des homosexuels sont devenus - avec cette rhétorique - un symbole de " civilisation » et, en même temps, une légitimation aux interventions «civilisatrices » dans les pays non occidentaux.

À l'inverse, dans le lieu où j'ai conduit ma recherche, les rapports sexuels entre personnes du même sexe étaient historiquement interprétés plutôt dans le cadre de la personnification dans le genre opposé au sexe conféré à la naissance. Cette performativité du genre garantissait la répétition de la règle hétéronormative et donc la stabilité du système. Il est important à ce propos de souligner que la performativité du genre opposé était beaucoup plus acceptée quand la personne quittait son rôle et son statut social masculin pour se rapprocher de la féminité. Les motivations à la base de ce raisonnement peuvent être identifiées grâce à la forte hiérarchie du genre et à l'importance symbolique (mais pas seulement) accordée à la supposée hégémonie masculine. Cette catégorisation des rapports homo-érotiques peut être analysée comme une des modalités sociales possibles pour soutenir la structure hétéronormative, la hiérarchie du genre et pour réduire considérablement le pouvoir subversif de la différence sexuelle et du genre (Mathieu, 1991).

Dans cette situation on peut donc affirmer que la dichotomisation entre hétérosexuel et homosexuel du point de vue identitaire est une nouveauté importée par la mondialisation et par l'internationalisation de la partie universaliste du mouvement LGBT occidental (Massad, 2007). Paradoxalement, dans ces milieux le développement d'une lutte LGBT fortement identitaire peut avoir pour effet d'augmenter l'homophobie, car elle produit des nouveaux sujets qui ne sont pas normalisés par le processus analytique de l'inversion du genre et qui se situent en dehors du système sexe-genre local.

À partir de cette analyse préliminaire, il est déjà évident que la situation du terrain, du point de vue du positionnement du chercheur, était suffisamment compliquée et concernait une grande variété d'acteurs sociaux. Si d'un côté les dangers liés à un hypothétique coming out du chercheur avec la plupart des interlocuteurs paraissent évidents, de l'autre la position «honnête » avec les militants LGBTIQ n'était pas si simple à soutenir.

Le mouvement pour les droits des minorités sexuelles en Afrique est fortement soutenu économiquement par les organisations LGBTI occidentales. Ces dernières sont aussi une source de légitimation d'une typologie de lutte qui paraît inspirée du mouvement homosexuel occidental (trouvant ses racines symboliques dans la célèbre révolte nord-américaine de Stonewall en 1969). Dans un environnement marqué par une forte homophobie, comme celui de mon terrain, l'Occident est perçu comme une source d'aide, de financement et de légitimation par une grande partie des militants LGBTIQ. Ma position politique et ma posture de recherche dans cette situation furent 
très critiques surtout au regard des mécanismes xénophobes, islamophobes, homonationalistes (Puar, 2007) et du pink-washing ${ }^{9}$ qui sont instrumentalisés pour légitimer les ingérences occidentales en Afrique et l'occultation de l'homophobie occidentale (Awondo et al., 2012). J'étais donc plutôt convaincue qu'il fallait essayer de ne pas être étiquetée par les militants LGBTIQ comme l'Occidentale qui arrive en Afrique pour "créer» le mouvement LGBTI, pour établir un pont avec les grandes associations européennes.

En conséquence de tout ça - au début de mon séjour sur le terrain - j’avais décidé de ne pas m'engager dans la lutte locale. Respecter cette décision n'a cependant pas été ni simple ni très réaliste dans la pratique quotidienne. De plus, la violence contre les homosexuels était toujours plus éclatante et angoissante, influençant ainsi mon rapport avec les militants, notre niveau d'empathie et de confiance.

\section{Devenir militant LGBTIQ sur le terrain : un choix chargé de conséquences}

La quotidienne réalité de la violence homophobe a bientôt remis en question mes convictions. Cette situation m'a conduit à réfléchir - à nouveau et de manière différente - à ma posture et à mon positionnement. Malgré le fait que les militants LGBTIQ de la ville étaient les personnes avec lesquelles j’avais développé le plus d'empathie, j'avais la perception d'être au dehors de «l'espace de la peur " (Green, 1994) et des stratégies politiques vécues et pensées par eux. C'est aussi pour cette raison que j'ai pris la décision de m'engager politiquement. Par conséquent, ma relation avec les militants changea énormément, d'abord concernant l'empathie, puis vis-à-vis des attentes liées à mon origine occidentale. En outre, le choix de l'activisme a produit de nombreuses conséquences dans ma relation avec la population hétérosexuelle de la ville et, en particulier, avec les leaders du discours homophobe. Avec eux le rapport était toujours influencé par la substantielle absence d'empathie et de sincérité. La seule idée que ces personnes puissent découvrir mon orientation sexuelle, mon lien avec les militants LGBTIQ ou le réel sujet de ma recherche de doctorat, me terrifiait.

Ma décision de m'engager dans la lutte contre l'homophobie a eu des effets sur ma recherche et m'a exposée aux dangers de la violence homophobe. J'étais devenue partiellement ou potentiellement - une cible de la violence. Contre toutes mes prévisions, ce changement radical de positionnement s'est pourtant révélé très productif d'un point de vue méthodologique.

\footnotetext{
${ }^{9}$ Le mot pinkwashing est utilisé pour décrire tous les épisodes dans lesquels certains États, entreprises et armées utilisent la promotion des droits des homosexuels comme stratégie de marketing pour déguiser d'autres formes de violations des droits de l'Homme.
} 
Loin d'une perception cynique de la violence, l'analyse conduite se focalise uniquement sur la question méthodologique et laisse donc de côté mes réactions et mes émotions face aux situations dangereuses. J’ai néanmoins conscience que mon exposition - potentielle ou réelle - à la violence fut toujours moins dangereuse que celle vécue par mes interlocuteurs de terrain. À ce propos, il ne faut jamais oublier le problème du privilège occidental afin de problématiser et contextualiser les limites structurelles de l'empathie, ainsi que la responsabilité de l'anthropologue par rapport aux interlocuteurs du terrain et son exposition moindre aux dangers.

En Occident, quand il est question d'engagement politique LGBTIQ, nous sommes habitués à imaginer une typologie de lutte très visible et publique. Au contraire, dans le cas dont il est question, les actions militantes sont souvent clandestines (ou partialement clandestines) et se concentrent sur la réduction des effets de l'homophobie. Il s'agit surtout de cacher les personnes encourant le risque d'être victimes de violence, de garantir l'accès aux services de santé (surtout en relation aux MST - maladies sexuellement transmissibles) et de construire, jour après jour, un parcours de mobilisation et de sensibilisation contre l'homophobie et d'intégration des personnes homosexuelles et transsexuelles. Donc en parlant de «devenir militante » je n'entends pas par là une sorte d'entrée officielle dans un espace public militant, mais plutôt un engagement quotidien dans une pratique politique clandestine.

Bien entendu, le choix de n'être pas visible est lié à la question de la dangerosité, mais aussi à une forme d'importance sociale de l'invisibilité. Un grand sujet de débat reste la différence entre pays africains et occidentaux sur la question LGBTIQ. Dans beaucoup de sociétés africaines et musulmanes, l'invisibilité des comportements sexuels non normatifs a, ou avait, une signification différente (Arnfred 2004; Murray, 1997) de l'invisibilité propre à la société occidentale qui la catégorise uniquement comme conséquence négative de l'homophobie.

Évidemment, cette clandestinité de l'activisme LGBTIQ m’a donné la possibilité de maintenir, pour quelque temps, le secret de mon engagement politique. Bien sûr, dans une petite ville, comme celle de mon terrain, il n'était pas possible de rester dans l'ombre. À cet égard, je trouve important d'analyser deux processus qui ont fortement conditionné ma relation avec mes interlocuteurs homophobes, suite à ma décision de devenir militant LGBTIQ: les jugements fondés sur ma provenance géopolitique d'une part, et les tentatives de contrôle générées par la peur de mon engagement dans l'activisme local d'autre part.

Un épisode en particulier est emblématique des jugements générés par ma provenance géopolitique. Suite à une agression, un homme avait été violemment tué. La victime étant bien connue pour être ouvertement homosexuelle, les militants LGBTIQ de la ville ont commencé à soupçonner une agression de nature homophobe. Avec d'autres militants, nous avons conduit des enquêtes sur l'homicide dans une atmosphère lourde de jugement et de soupçons. Le jour après le début de notre enquête un militant LGBTIQ de la ville reçut notamment un message anonyme. Le message était 
assez clair : s'il ne voulait pas subir le même sort, il devait abandonner les enquêtes et plus en particulier il ne devait pas les conduire avec « la fille des droits de l'Homme »c'est-à-dire, moi.

Je ne m'étais toutefois jamais présentée en tant que promotrice des droits de l'Homme. Le sujet ici était plutôt le rappel à ce langage universaliste dû à mon origine occidentale. En Afrique les droits de l'Homme sont en effet perçus par la plupart de la population comme une redoutable source de pouvoir, comme le symbole du nouveau projet civilisateur des pays occidentaux. Sur mon terrain, l'évidence du rôle de l'Occident comme sujet désiré, désirable, détesté, combattu et en tant que présence symboliquement et politiquement très importante est devenue frappante à partir du moment où je me suis engagée politiquement. J'ai ainsi pu observer la réaction locale à la présence d'une femme occidentale penchée sur la question des minorités sexuelles.

Une autre question intéressante est celle de mon exposition à la violence physique et psychologique que j'ai interprétée et analysée comme le résultat d'une tentative de contrôle sur mes actions. À l'époque de mon engagement avec les militants LGBTIQ, je poursuivais les contacts et les relations de terrain avec la population hétérosexuelle de la ville et je réalisais beaucoup d'entretiens avec les leaders du discours homophobe. Ce double parcours méthodologique m'a aidé à enquêter sur le rapport entre "homosexuels» et "homophobes", ainsi que sur les représentations symboliques produites par cette relation, avec une attention particulière aux liens entre intimité, violence et sexualité.

Le complexe rapport entre violence et proximité, séduction et répulsion est devenu plus clair quand j'ai réalisé un troisième entretien avec l'un de mes enquêtés. Grâce à certaines rumeurs mêlées à mon enquête, j’avais découvert qu'il cultivait des relations sexuelles clandestines avec d'autres hommes. En même temps, il était en fait déjà au courant de mon engagement politique. Dès mon arrivée chez lui, j’ai compris que j'étais en train de subir une agression : il était très nerveux et son attitude n'était pas du tout conforme à l'hospitalité locale à laquelle j'étais habituée. Il a même commencé à me menacer et m'apostropher en tant qu'« amie des homosexuels » et "Occidentale ». La situation devint aussi intéressante qu'angoissante lorsque l'homme passa de cette accusation agressive à une tentative de séduction. Je me retrouvai entre l'un et l'autre, essayant d'arrêter son comportement schizophrénique lorsqu'il me frappa d'un coup de poing dans le ventre en affirmant qu'il tuerait « mes amis homosexuels ». Je décidai à ce moment-là de m'échapper très rapidement de la maison. Même si cet épisode fut difficile et lourd à supporter, je pus observer de cette manière d'un peu plus près une partie du rapport très ambivalent entre séduction, agression, violence et revendication qui prend corps dans la lutte locale de l'activisme LGBTIQ, ainsi que les tentatives de contrôle sur les actions des acteurs sociaux menées par ces personnes. 


\section{Conclusion}

Les deux épisodes évoqués précédemment expliquent bien les motivations profondes qui sous-tendent mes réflexions sur mon terrain : mon positionnement comme chercheuse occidentale, lesbienne et engagée dans la lutte pour les droits des minorités sexuelles constitua une source méthodologique extrêmement intéressante. Bien que les enjeux de la rhétorique autour des droits de l'Homme aient déjà été étudiés en lien avec la construction du discours universaliste occidental et ses effets structurels (Massad, 2007), il existe très peu d'instruments disponibles pour analyser ce même problème au niveau de l'imaginaire local. Par le biais des menaces reçues je me suis rendue compte que ma présence dans le milieu militant était perçue - par certaines personnes - comme directement liée à la lutte pour les droits de l'Homme. J'ai mieux compris l'origine de leur représentation en tant que force terrible, incontrôlée, «blanche» et complètement étrangère à la construction du discours local sur les droits. Après la menace reçue, je me suis rappelée que, pendant un de nos entretiens, un activiste avait utilisé la phrase « Maintenant il y a les droits de l'Homme » comme menace contre des gens qui ne veulent pas collaborer avec les enquêteurs. Tout se passait comme si les droits de l'Homme étaient une arme utilisée pour rappeler un imaginaire de peur et d'obscurité.

De la même façon, l'agression dont je fus victime m'a permis de comprendre à la fois que l'homophobie peut être enclavée dans le continuum émotionnel se déployant entre séduction, désir, peur et répulsion, et que la sexualité est une arme utilisée pour la négociation des relations de pouvoir. Malgré mon choix initial de ne pas révéler mon orientation sexuelle et suite à mon engagement dans la lutte locale, les changements méthodologiques provoqués par le développement de mon terrain ont donc été particulièrement intéressants. J'ai voulu souligner comment mon exposition à des épisodes de violence m’a amenée à découvrir de nouvelles pistes de recherche. Si une grande partie de mon élaboration théorique dans la thèse est effectivement fondée sur une critique radicale des processus néo-colonialistes occidentaux en Afrique avec une attention particulière à la question de l'universalisme des droits de l'Homme pour les minorités sexuelles -, d'un autre côté j'ai analysé la violence comme le résultat des liens entre désir et répulsion au sein des rapports homo-érotiques. Dans ce contexte, l'enjeu n'est pas de simplifier le problème de la violence en le ramenant à un niveau individuel, mais plutôt de comprendre comment l'homophobie structurelle génère des formes de souffrance psychologique graves au point de dégénérer en épisodes de violence psychique. L'agression à laquelle je fus exposée est particulièrement utile pour ouvrir l'analyse à la relation entre séduction et répulsion ainsi qu'aux modalités de contrôle que violence et sexualité ont sur les corps et les comportements sociaux.

Bien qu'ayant essayé d'éviter le plus possible d'être exposée à la violence, j’ai trouvé ces épisodes très utiles en tant que source d'informations. Si l'ethnographie est un 
parcours fait de rencontres, il ne peut pas être seulement fait d'empathie et de résonances émotives. Il y a toujours des moments de difficulté dans le travail de terrain, des zones obscures et des relations conflictuelles. L'enjeu pour moi était de travailler de manière réflexive sur le rapport entre sujet de recherche, chercheur, interlocuteurs de terrain et violence. La recherche nécessite d'approfondir la thématique de la violence, mais chercheur et interlocuteurs de terrain doivent dans le même temps se protéger de cette violence. Par ailleurs, si le chercheur provient d'un contexte culturel et social différent, il doit tenir compte du fait qu'il n'est pas capable de prévoir les conséquences de ses actions (Peritore, 1990). Il est aussi impossible dans cette situation de se préserver au dehors de la violence, il est donc important de problématiser la question en évitant tout autant d'être terrifié que de se sentir anesthésié et de perdre la violence comme variable d'étude (Green, 1994). Bien sûr, d'un point de vue méthodologique la question n'est pas facile à résoudre et il est impossible de faire une théorisation au-dehors du contexte spécifique du terrain. Le point important ici est de considérer que l'entrée dans certains mécanismes de reproduction symbolique de la violence peut constituer un point de réflexion important concernant le terrain.

En conclusion de ces réflexions, trois éléments doivent être mis en évidence pour comprendre mon ajustement et ma proximité avec la violence homophobe : le privilège occidental, l'aide et la complicité de mes amies activistes LGBTIQ locales pour obtenir des informations et trouver des stratégies pour échapper aux agressions, et enfin le statut de chercheuse universitaire. Ces trois éléments m'ont permis d'être proche et spectatrice de la violence, mais sans jamais souffrir de réelles conséquences de l'expression de cette violence. Comme soutenu par Donna Haraway (1991), « $a$ commitment to mobile positioning and to passionate detachment is dependent on the impossibility of innocent "identity" politics and epistemologies as strategies for seeing from the standpoints of the subjugated in order to see well. [...] Vision is always a question of the power to see - and perhaps of the violence implicit in our visualizing practices » (p. 192). La problématisation explicite du privilège de l'anthropologue occidentale dans un contexte postcolonial doit, à mon avis, être toujours considérée avec attention.

\section{Bibliographie}

ABu-Lughod L. (2006), "Writing against Culture», E. LeWIN (dir.), Feminist Anthropology: A Reader, Malden, Blackwell, p. 153-169.

ARNFRED S. (dir.) (2004), Re-Thinking Sexualities in Africa, Uppsala, Nordiska Afrikainstitutet.

Awondo P., Geschiere P., Reid G. (2012), «Homophobic Africa?: Toward a more nuanced view », Africa Studies Review, vol. 55, n 3, p. 145-168.

BRoQuA C. (2015), «Les pro, les anti et l'international: mobilisations autour de l'homosexualité en Afrique de l'Ouest», in K. Tall, M-E. Pommerolle, M. Cahen 
(dir.), Collective Mobilisations in Africa / Mobilisations collectives en Afrique, Leyde, Boston, Brill, p. 183-204.

BUtLER J. (1990), Gender Trouble: Feminism and the Subversion of Identity, New York, Routledge.

BuTLER J. (2004), Undoing Gender, New-York, Londres, Routledge.

Connell R. W., Messerschmidt J. W. (2005), « Hegemonic Masculinity. Rethinking the Concept », Gender Society, vol. 19, n 6, p. 829-859.

Epprecht M. (2008), Heterosexual Africa? The History of an Idea from the Age of Exploration to the Age of AIDS, Athens, Ohio University Press/University of KwaZulu-Natal Press.

EVANS-PRITCHARD E. E. (1970), "Sexual Inversion among the Azande», American Anthropologist, vol. 72, $\mathrm{n}^{\circ}$ 6, p. 1428-1434.

GREEN L. (1994), « Fear as a way of life », Cultural Anthropology, vol. 9, n² 2, p. 227-256.

Haraway D. J. (1991), Simians, Cyborgs, and Women. The Reinvention of Nature, New York, Routledge.

Herek G. M. (2000), "The Psychology of Sexual Prejudice», Current Directions in Psychological Sciences, vol. 9, n 1, p. 19-22.

HOAD N. W. (2007), African Intimacies. Race, Homosexuality and Globalization, Minneapolis, Londres, University of Minnesota Press.

MASSAd J. (2007), Desiring Arabs, Chicago, Londres, The University of Chicago Press.

Mathieu N.-C. (1991), L'anatomie politique. Catégorisations et idéologies du sexe, Paris, Côté-femmes éditions.

Morgan R., Wieringa S. (2005), Tommy Boys, Lesbian men and ancestral wives. Female same-sex practices in Africa, Johannesburg, Jacana Media.

Murray S. O. (1997), "The Will Not to Know. Islamic Accomodations of Male Homosexuality », S. O. Murray, W. Roscoe, Islamic Homosexualities. Culture, History and Literature, New York, Londres, New York University Press, p. 14-54.

Murray S. O., Roscoe W. (1998), Boy-wives and female husbands. Studies on African homosexualities, New York, Palgrave.

Peritore N. P. (1990), « Reflections of Dangerous Fieldwork», The American Sociologist, vol. 21, n 4, p. 359-372.

PUAR J. K. (2007), Terrorist Assemblages. Homonationalism in queer times, Durham, Duke University Press. 
RuBiN G. (2006), «The Traffic in Women: Notes on the "Political Econominy" of Sex », E. LeWIN (dir.), Feminist Anthropology: A reader, Malden, Blackwell, p. 87- 106.

TAUssig M. (1986), Shamanism, Colonialism and the wild man: a study in terror and healing, Chicago, Londres, University of Chicago Press.

WeEKS J. (1987), « Questions of identity », in P. CAPLAN (dir.), The Cultural Construction of Sexuality, New York, Routledge, p. 31-51.

Weinberg G. H. (1972), Society and the healthy homosexual, New York, St. Martin's Press. 